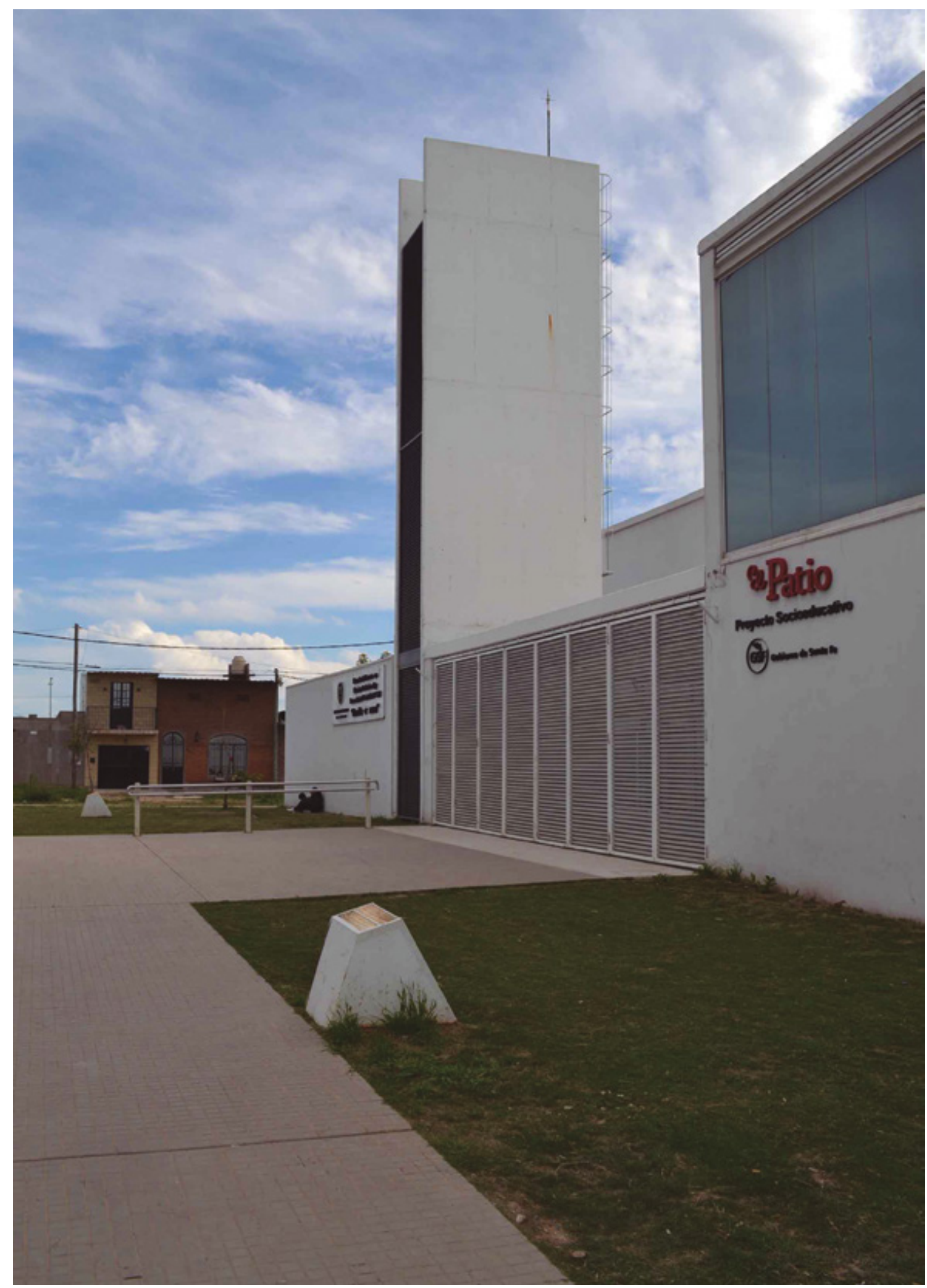

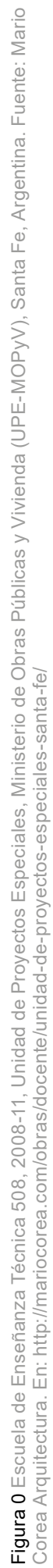



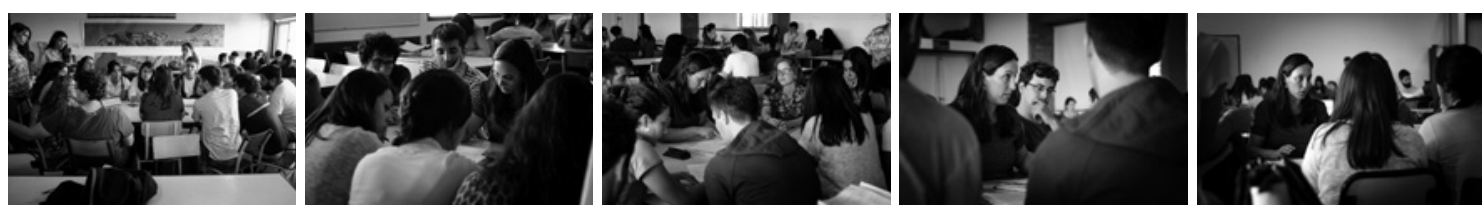

Secuencia: Clase de Teoría y Crítica en FADU, UNL

Fotos: Germán Godoy Hernández

\title{
ESCUELAS: TRADICIÓN PROYECTUAL EN ARQUITECTURA PÚBLICA EN ARGENTINA'
}

\author{
SCHOOLS: THE PROJECT TRADITION IN PUBLIC ARCHITECTURE IN ARGENTINA'
}

\section{Lucía Espinoza²}

\section{RESUMEN}

La arquitectura escolar, sus problemas y sus fórmulas, funcionan como una constante de la arquitectura pública en la Argentina moderna, configurando una suerte de tradición proyectual que se reedita en cada proyecto. Se reconoce en el final del siglo XIX, un primer momento fundante de criterios y procedimientos que determina un valor central y un rol instrumental para la arquitectura producida desde el Estado. Y, también, un segundo momento situado a finales del siglo $X X$, que altera las condiciones culturales y técnicas del ejercicio profesional desde la estructura del Estado y diversifica los modos de elaboración del proyecto arquitectónico. Uno de los objetivos de esta investigación es la identificación de las tensiones contemporáneas que asume la tradición proyectual que asocia arquitectura escolar y políticas públicas, localizadas en marcos explicativos e interpretativos que incluyan la trama histórica.

Palabras clave: arquitectura escolar, arquitectos, historia, obras públicas, Argentina.

\section{ABSTRACT}

School architecture, its problems and its formulas, function as a constant of public architecture in modern Argentina, and have formed a kind of project tradition that is reedited in each new project. A first founding moment is recognized to have occurred at end of the nineteenth century, in which criteria and procedures established the core value and instrumental role of state-produced architecture. A second moment took place at the end of the twentieth century, which altered the cultural and technical conditions of professional practice based on the structure of the state, and diversified the ways of creating architectural projects. One of the objectives of this research is to identify the contemporary tensions that the project tradition has taken on, which associate school architecture and public policies, located in explanatory and interpretative frameworks that take into account the fabric of history.

Keywords: school architecture, architects, history, public works, Argentina.

Artículo recibido el 26 de junio de 2017 y aceptado el 11 de noviembre de 2017 DOI: https://doi.org/10.22320/07196466.2017.35.052.05

[1] Este artículo se basa en los resultados de dos proyectos de investigación: CAI+D 2011: Arquitectura y ciudad en los proyectos recientes del Estado. Registros en Santa Fe, 2007-2011, dirección: Lucía Espinoza (código PI: 50120110100267, FADU, UNL, Argentina, 2013-2016); y tesis doctoral: Arquitectura educativa y políticas públicas en Santa Fe (2007-2011). Producción y comunicación de la arquitectura en el Estado, autora Lucía Espinoza, dirección: Roberto Fernández, co-dirección: Bibiana Cicutti (Doctorado en Arquitectura, FAPyD, UNR, Argentina, 2012-2016).

[2] Facultad de Arquitectura Diseño y Urbanismo Universidad Nacional del Litoral, Santa Fé Argentina. Iucia_eb@hotmail.com.ar 
La arquitectura escolar funciona como una constante de la arquitectura -en cuanto disciplina- en la Argentina moderna. Desde la conformación de las instituciones del Estado a fines del siglo XIX, hasta la reforma estructural del último tramo del siglo $\mathrm{XX}$, los edificios escolares, junto a los desafíos que afronta la arquitectura pública en cada segmento histórico, se posicionaron como temática específica dando inicio a una tradición proyectual $^{3}$ basada en una serie de problemas que se reeditan en cada proyecto.

Los problemas que versan en torno a los edificios escolares son: discontinuidad entre la arquitectura y la ciudad; representación institucional y modalidad de producción burocrática basada en la confianza en el saber disciplinar y en su especialización temática (Espinoza, 2016: 287-317). Para abordar estas problemáticas proyectuales se fueron configurando fórmulas también ancladas en la memoria disciplinar: el aula como unidad compositiva o módulo del proyecto; el patio central de geometría regular asociado al control disciplinar; la composición arquitectónica basada en la organización jerárquica del programa pedagógico; la concentración de los aspectos simbólicos del lenguaje en el acceso al edificio y como frase principal de la sintaxis para la representación institucional, entre las soluciones más canonizadas (Figura 1 y 2).

Así, problemas y fórmulas se repiten y se combinan según las tendencias o las preocupaciones históricas de la disciplina, dependiendo igualmente de las políticas pedagógicas y de la importancia que les asignen a estos temas. Por eso, las gestiones de gobierno se consideran núcleos para la interpretación de las políticas públicas, tanto en infraestructura, como en educación.

Este trabajo sostiene que esa continuidad en el valor dado al proyecto de arquitectura pública-escolar en Argentina, que se institucionaliza con la Ley 1.420 (1884) en las oficinas técnicas del Consejo Nacional de Educación (CNE) y se manifiesta incluso durante la dictadura militar en un plan de escuelas municipales en Buenos Aires (1979) (Figura 3), es reformulada en la última década del siglo en un contexto histórico que altera las condiciones culturales y técnicas del ejercicio profesional en el Estado y diversifica los modos de elaboración del proyecto arquitectónico.

En este contexto, el documento Criterios y Normativa Básica de Arquitectura Escolar (Ministerio de Cultura y Educación de la Nación, 1998) elaborado para regular las obras necesarias para la implementación del sistema educativo dispuesto por la Ley Federal de Educación (1993), adquiere relevancia en la medida en que establece las pautas y consolida los procedimientos que condicionan las operaciones proyectuales sobre escuelas hasta el presente. 


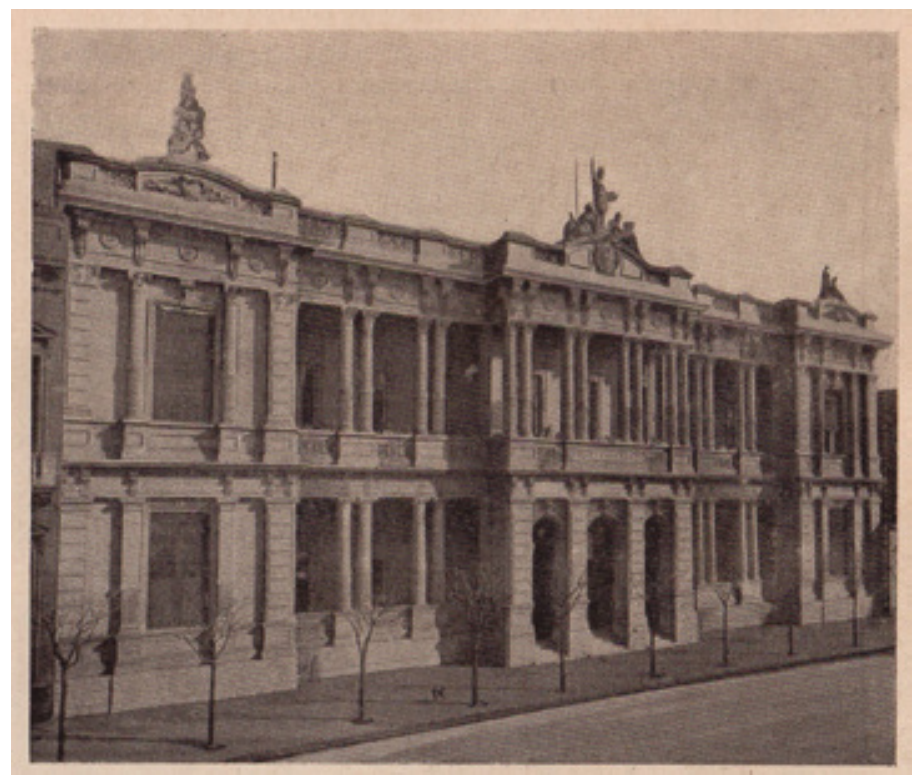

Figura 1 Escuela Sarmiento, fachada, 1886, Arqs. Carlos Morray R. Batlle, Consejo Nacional de Educación, Buenos Aires, Argentina. Fuente: La Historia Argentina de los niños en cuadros. Buenos Aires: Edición del Centenario, Lajouane y Ca. editores, 1910. p. 12

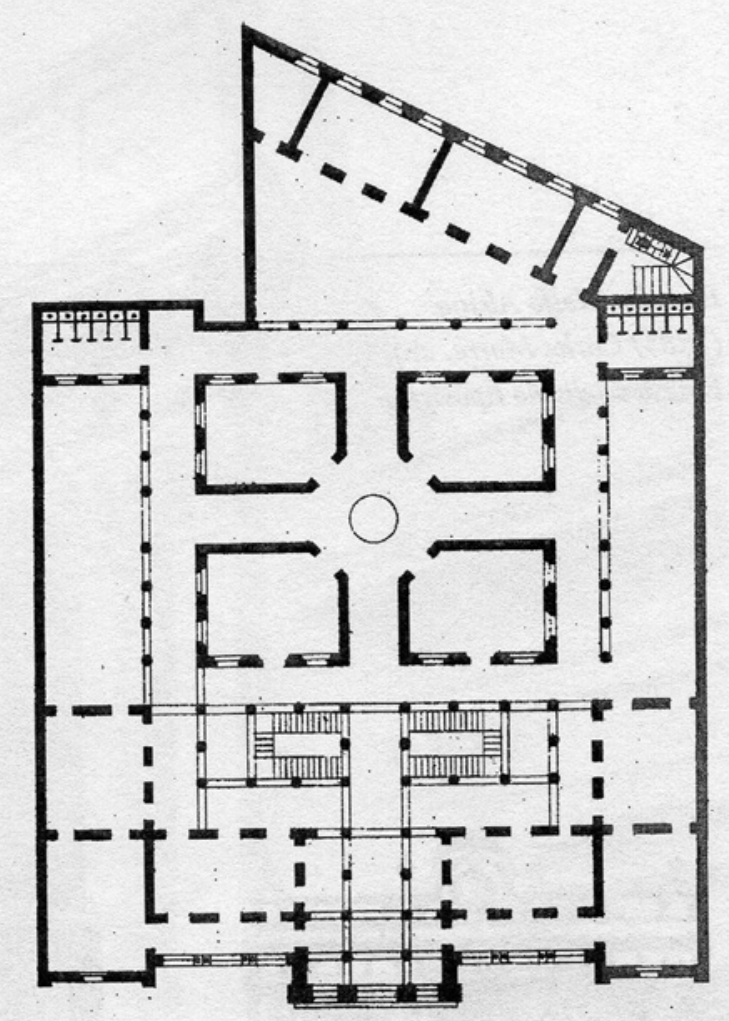

Figura 2 Escuela Sarmiento, planta baja, 1886, Arqs. Carlos Morra y R. Batlle, Consejo Nacional de Educación, Buenos Aires, Argentina. Fuente: Brandariz, G. La arquitectura escolar de inspiración sarmientina, Buenos Aires: Eudeba, 1998, p. 51 


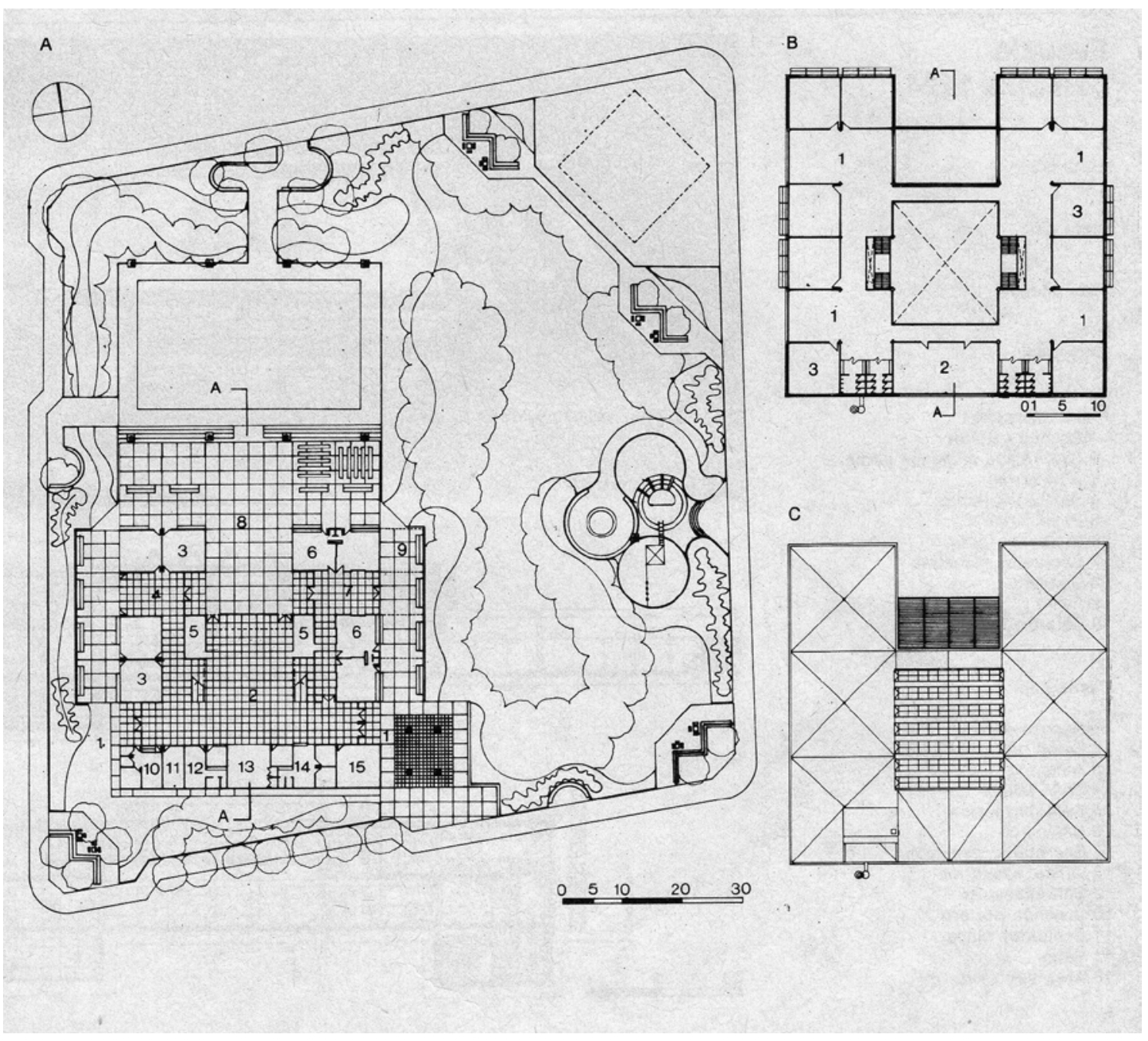

Figura 3 Prototipo del plan de primarias municipales, Coordinador general del Equipo de proyecto: Arq. Miguel Cangiano, 1979, Buenos Aires, Argentina. Fuente: Summa145/146, Buenos Aires, enero/febrero 1980, p.87

\section{MÉTODOS}

El objetivo de este estudio es el reconocimiento de la tradición proyectual que asocia arquitectura escolar y políticas públicas en Argentina, desde la sanción de la Ley 1.420 en adelante, y la individualización de las tensiones contemporáneas que ésta asume.

Con dicho fin, se analizan episodios de arquitectura escolar a lo largo del siglo XX, seleccionados como índices para argumentar la existencia de un cuerpo de saber configurado en un segmento histórico que determina los agentes involucrados, define los problemas y las fórmulas arquitectónicas, los sistematiza y posibilita su transmisión en el tiempo. La selección de los casos (planes, obras y documentos normativos) remite a momentos clave para considerar la inercia y la reproducción de esta tradición proyectual, mediada por las tensiones históricas de cada contexto. Pero también, la diver- sidad de las experiencias se incluye para visibilizar una multiplicidad de variables que participan en la relación entre arquitectura, educación y política.

A contramano de una tendencia histórica, el trabajo busca producir parámetros de valorización enhebrando fragmentos de la historia de la arquitectura escolar, para inscribirlos en una trama de sentido ampliada que evidencie contrastes entre aparentes continuidades o series arquitectónicas homogéneas. En este sentido, se considera que uno de los aportes de esta propuesta es la incorporación de casos que permiten abordar la condición contemporánea de la arquitectura escolar en Argentina en el contexto de una trama histórica.

La mayor dificultad en el desarrollo de la investigación sobre el pasado reciente es la construcción de las bases de datos sobre obras, estructuras técnicas y agentes. La cantidad y la diversidad de las fuentes en las que se difunde información sobre obra pública, incluso dentro 
de las órbitas oficiales, complejizan el acceso a datos certeros y obligan a trabajar con riguroso juicio crítico en cada instancia. En todos los casos, esta investigación prioriza los datos oficiales.

Como enfoque metodológico frente al análisis de una situación contemporánea en arquitectura, se elude la presentificación en las interpretaciones de los fenómenos para, en cambio, remitir a las tensiones históricas que atraviesan el presente.

\section{RESULTADOS}

En la Argentina, la historia de la arquitectura escolar está asociada a su devenir como recurso de la política. Desde la creación del sistema educativo nacional, el problema del edificio escolar se instala en la agenda estatal con participación protagónica de la arquitectura y la consecuente intervención de sus debates centrales.

En la estructura estatal diseñada, el CNE proyecta las escuelas primarias y el Poder Ejecutivo, desde la Dirección de Obras Públicas, tiene a cargo las normales. Esta determinación que enlaza una serie de políticas públicas en sintonía con el objetivo de implementar el primer sistema educativo nacional, tiene carácter fundacional en relación al rol instrumental asignado a la arquitectura. Así, mientras el edificio escolar irrumpe como problema, la arquitectura se posiciona como componente estratégica del proceso de modernización, con la mediación de los arquitectos como agentes principales. Queda diseñada una plataforma de partida basada en la instrumentalización de la arquitectura, pero que también avanza en la definición de modalidades operativas, modelos y fórmulas proyectuales que, en adelante, pulsan como constantes históricas en contextos y circunstancias diferentes.

Los primeros edificios para escuelas, entre 1884 y 1910 en Buenos Aires, se debatían entre adoptar los modelos tipológicos del petit hôtel o del palacio y la necesidad de construir una imagen institucional homogénea y laica, a partir de la utilización tanto de prototipos como de modelos templarios (Gizzarelli, 1985; Brandariz, 1998; Shmitd, 2004).

En provincias como Santa Fe o Tucumán los primeros edificios escolares estatales se proyectan sobre el inicio del siglo y resultan de esquemas que asimilaron aquel debate y se configuraron a partir de la selección de modelos tipológicos completos o fragmentados, pero que nunca abandonaron la voluntad palaciega de la representación ${ }^{4}$ (Espinoza, 2005; Murillo Dasso, 2012).

En contraste, durante las décadas de 1930 y 1940, las escuelas que adoptan los códigos expresivos de la arquitectura moderna en las provincias centrales, coinciden en abrir la discusión confrontando con los modelos ar- quitectónicos implementados desde las oficinas técnicas nacionales. Pero, para reconocer los sentidos que asumen las propuestas de renovación del lenguaje arquitectónico en Mendoza, Córdoba, Santa Fe o Tucumán, se requiere estudiar las políticas provinciales y sus articulaciones con la órbita nacional, ya que se establecen diferencias que hacen aportes sustanciales en la trama interpretativa de estas decisiones proyectuales. Por ejemplo, entre 1932 y 1943, los casos de Santa Fe y Mendoza aportan elementos para una lectura comparada de estos procesos (Raffa y Espinoza, 2013; Cattaneo, 2015). La producción arquitectónica santafesina en clave modernizante trasciende los cambios de signo político sucedidos en las gestiones provinciales. Las escuelas modernistas proyectadas por la Dirección de Obras Públicas (DOP) avanzan en la experimentación formal y ensayan opciones tipológicas abiertas: "en cruz", compartiendo una plaza con el barrio, o bien, provocando usos comunitarios en el programa escolar como la incorporación de una biblioteca popular o baños públicos en instituciones ubicadas frente a parques, que buscan ajustarse a las tendencias pedagógicas modernas en el marco educativo reformista del gobierno

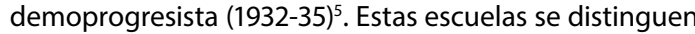
como dispositivos de modernización por el modo en el que irrumpen con la estética de la abstracción en el paisaje incompleto de los barrios que forman el crecimiento extensivo de las ciudades o en el territorio de apropiación dispersa que caracteriza a los pueblos de provincia (Figuras $4,5,6$ y 7 ).

Sin embargo, las políticas reformistas se interrumpen con la intervención nacional de 1935 y concluyen con el gobierno conservador (1937-41). Lo que interesa subrayar aquí es que la continuidad en las búsquedas de renovación del lenguaje arquitectónico se da a pesar del cambio radical en la orientación de las políticas educativas y se explica por razones de naturaleza distinta pero que se vinculan en el análisis. A saber, por la manifestación de una autonomía del lenguaje modernizante como opción disciplinar respecto de las políticas públicas -ambas modernizadoras: reformistas o conservadoras-; e, igualmente, por la obra de una oficina técnica que tiene continuidad en sucesivas gestiones de gobierno con un mismo equipo de profesionales. El trabajo del cuerpo técnico de la DOP asegura encadenamiento en las premisas y en los modos de elaboración del proyecto en la producción de arquitectura escolar santafesina $y$, a la vez, acumula experiencia transferible en la materia (Espinoza, 2005). En esta dirección, reconociendo una gestión en obra pública a partir de la continuidad de los agentes, es que se explica la experiencia del mismo periodo en Mendoza. Pero, a diferencia del caso santafesino, la continuidad de un mismo sector político en el gobierno durante toda la década está acompañada por la unificación de la gestión de la obra pública en la conducción de los arquitectos $\mathrm{Ci}$ vit. Desde esta perspectiva, las condiciones particulares y las características de cada contexto provincial, distinguen y multiplican las referencias para analizar las circunstancias ambiguas en las que se configuran los procesos de modernización disciplinar (Gorelik, 2003). 


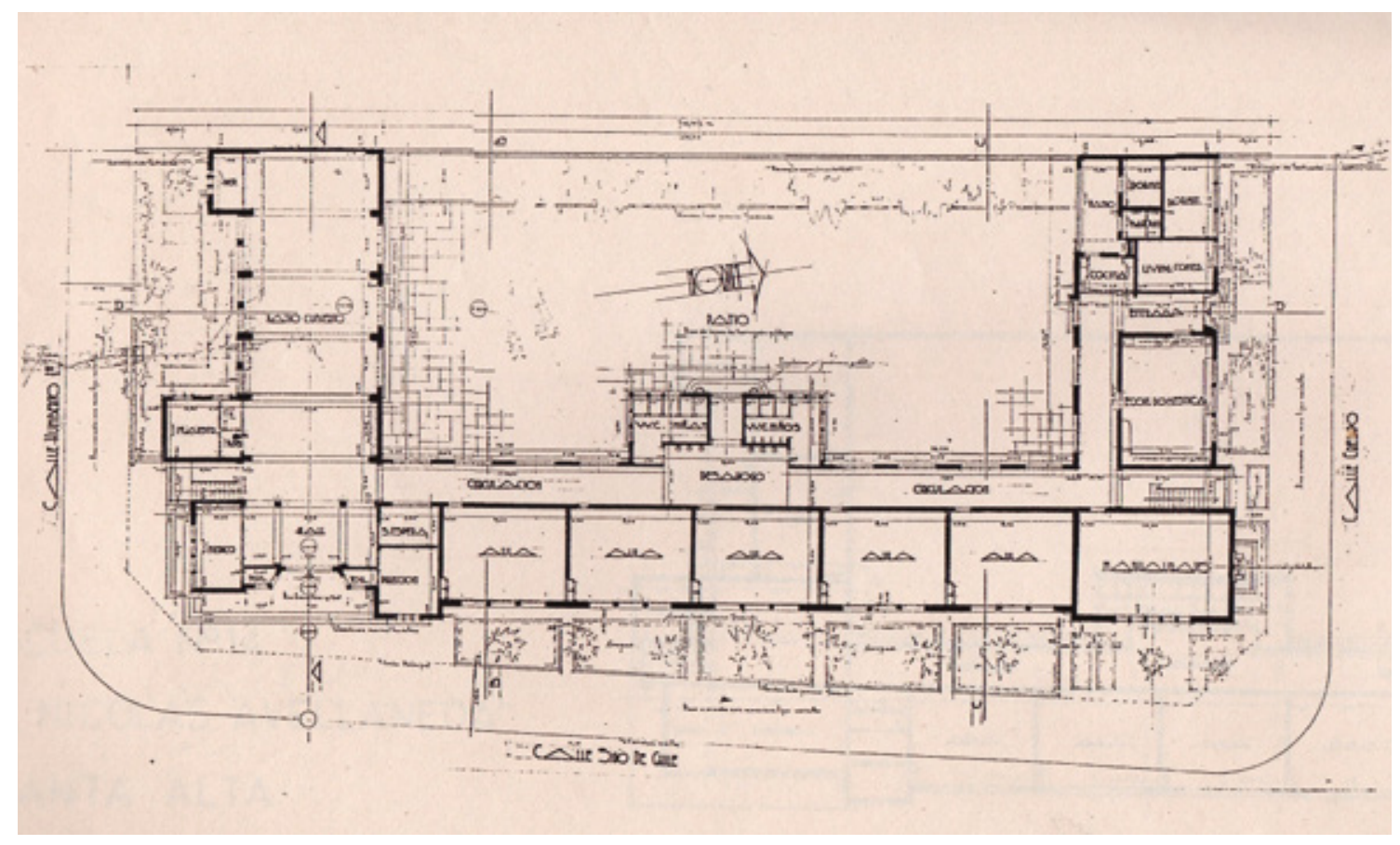

Figura 4 Escuela primaria Nicolás Avellaneda, planta baja, 1940, Arq. Croci, Dirección de Obras Públicas, Ministerio de Obras Públicas (DOP, MOP), Santa Fe, Argentina. Fuente: Archivo Instituto de Teoría e Historia Urbano-Arquitectónica (INTHUAR), FADU, UNL

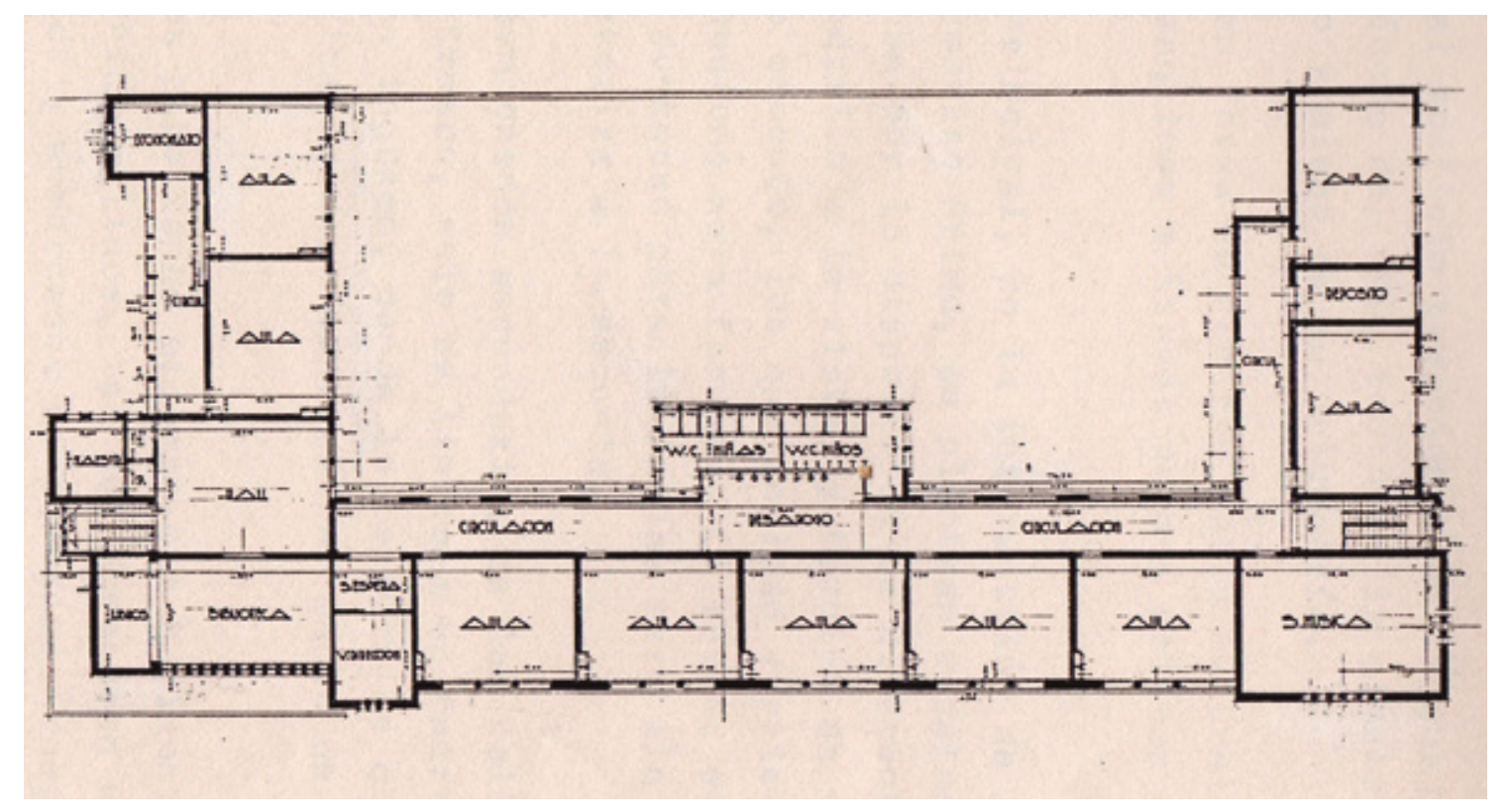

Figura 5 Escuela primaria Nicolás Avellaneda, planta alta, 1940, Arq. Croci, DOP, MOP, Santa Fe, Argentina. Fuente: Archivo INTHUAR, FADU, UNL 


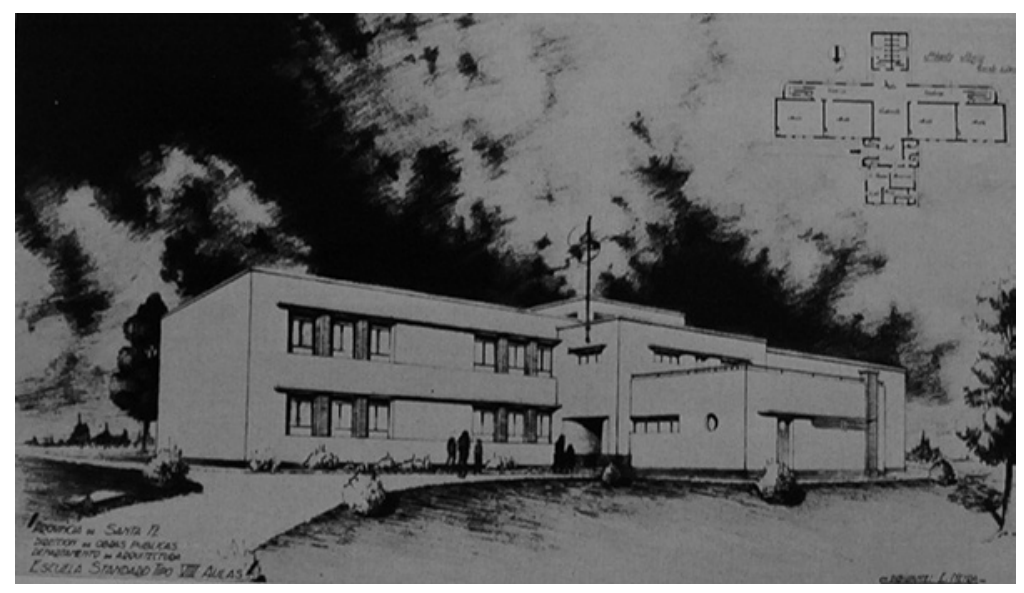

Figura 6 Proyecto de Escuela Standard, tipo 8 aulas, 1938, DOP, MOP, Santa Fe, Argentina. Fuente: Boletín de Obras Públicas de la República Argentina, 42, 1938, p. 71

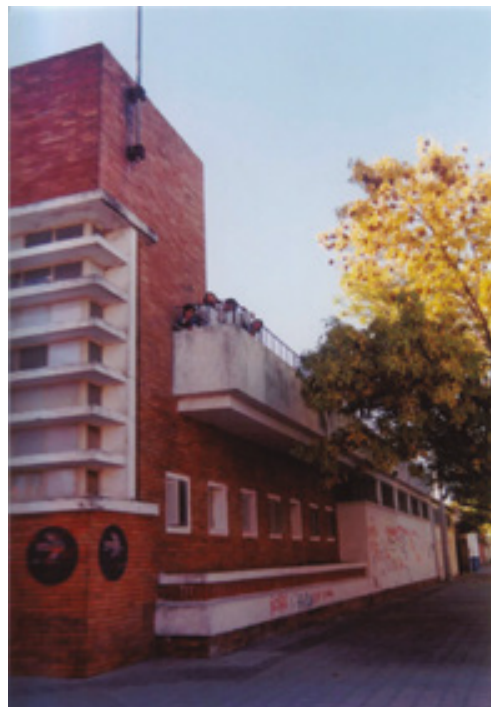

Figura 7 Escuela primaria Juan Arzeno, 1940. Arq. Croci, DOP, MOP, Santa Fe, Argentina. Fotografía: Lucía Espinoza

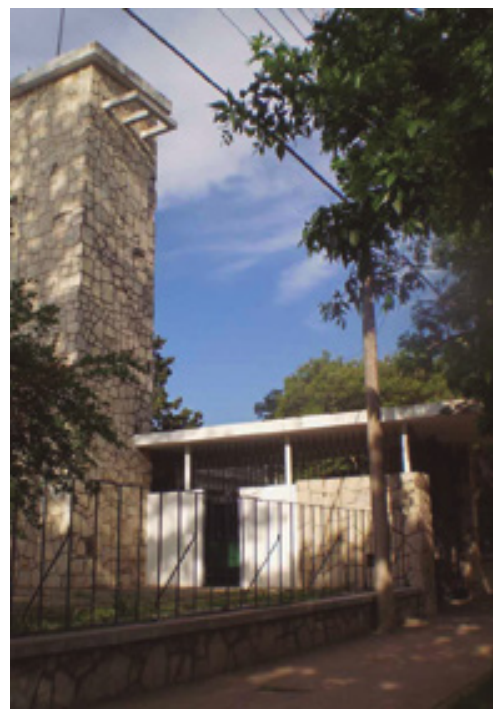

Figura 8 Escuela primaria Pascual Echagüe, 1950-55 MOP Santa Fe, Argentina. Fotografía: Lucía Espinoza

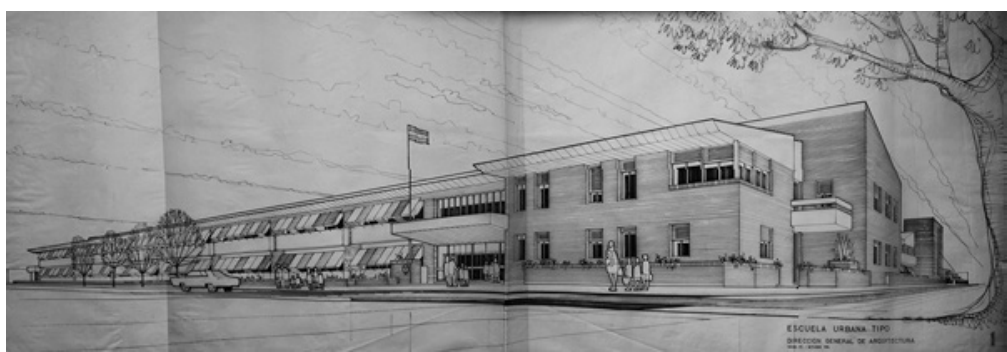

Figura 9 Escuela tipo urbana, 1976, Dirección General de Arquitectura, MOP, Santa Fe Fuente: Archivo DIPAI, MOP, Santa Fe 
A mediados del siglo $\mathrm{XX}$, estas experiencias se ponen en contacto con la política de centralización de las decisiones y de la tematización expresiva de las escuelas construidas por el primer peronismo. Otra vez se recurre a la renovación del lenguaje para identificar una gestión de gobierno junto a la definición de un método proyectual basado en la elaboración de planos tipo para configurar y reproducir modelos, versionados según la región y el programa escolar de referencia. Los cuerpos técnicos reestructurados en las provincias asumen el mandato nacional: intervienen ajustando los prototipos a las demandas de cada barrio o a las particularidades del lote elegido, pero siempre manteniendo la consigna de la unificación del lenguaje en clave rústica, como versión expresiva asumida para el programa escolar dentro del amplio repertorio que caracteriza a la arquitectura oficial peronista. Asimismo, se reconocen derivas de los modelos nacionales formuladas en las oficinas locales, sobre todo cuando los recursos técnicos profesionales atraviesan gestiones políticas y configuran un factor formador de masa crítica, reducida en su alcance operativo, pero que forma parte del mecanismo productivo burocrático como factor determinante en un margen variable de las decisiones proyectuales (Espinoza, 2011; Durá Gúrpide, 2017) (Figura 8).

Por su parte, las décadas de 1960 y 1970 protagonizan discusiones que trascienden la temática escolar, como las políticas de participación social y la profesionalización de los recursos técnicos estatales, que coexisten con las referencias determinantes del campo disciplinar como la metodología basada en los sistemas o la tendencia hacia la estética del hormigón visto, imprimiendo singularidad expresiva y autonomía a las obras producidas en el periodo. Y, si bien se reconocen casos emblemáticos en los cuales se reúnen condiciones como una complicidad coyuntural con los avatares contemporáneos de la pedagogía ${ }^{6}$, la producción escolar queda determinada por cierta independencia que adquieren los cuerpos técnicos estatales y su participación en los debates disciplinares (Figura 9).

Con posterioridad al retorno de la democracia, las sucesivas reformas en las estructuras del Estado afectaron la configuración de los cuerpos técnicos y de sus modalidades operativas. Para pensar el presente histórico, se sostiene la necesidad de remitirse a las transformaciones realizadas en la década del noventa (1989-2001) considerada como un segundo momento fundante de criterios y procedimientos. Los criterios subsisten en los documentos normativos y las metodologías están afianzadas en las prácticas arquitectónicas que definen la obra pública contemporánea, independientemente si se interviene desde el interior o no, de una estructura técnica estatal.

Pese a que el proceso reformista de fin de siglo registra un progreso en el vínculo entre arquitectura y pedagogía, la producción queda subsumida en los criterios de eficiencia y racionalización de los recursos que lideran el pensamiento finisecular. La sanción de la Ley Federal de Educación y sus requerimientos pedagógicos y espaciales, entre los que se distingue la incorporación de las tecnologías de la información y la comunicación al proceso educativo, determinan un programa específico para la arquitectura escolar: segmentación del edificio en niveles educativos, incorporación de aulas y sanitarios para albergar octavo y noveno año en las ex primarias, y agregado de aulas de informática en todas las escuelas. En el marco de esta política educativa nacional, se privilegian las operaciones de ampliación y sectorización de los edificios existentes que se realizan bajo la impronta normativa de Criterios y Normativa Básica de Arquitectura Escolar y según las demandas del programa de financiamiento involucrado (Plan Social y/o Pacto Federal Educativo), previo entrenamiento del cuerpo técnico reestructurado en Unidades creadas ad hoc en cada provincia. Los equipos técnicos alteran su tradicional organización de sectorización funcional -educación, salud, obras públicas- y se reordenan según una nueva estructura de "unidades" que establece jerarquías basadas en los programas de financiamiento e incluye la renovación generacional de los agentes.

El objetivo que instala la estructura técnica de diseño finisecular persigue la viabilidad financiera a partir de la aprobación de los proyectos de arquitectura. Se trata de operaciones técnicas de fuerte sentido político en el interior de la tradición proyectual analizada, ya que resitúan la concepción del proyecto de arquitectura en clave instrumental a partir de un procedimiento de control basado en indicaciones prolijamente fijadas en los manuales operativos diseñados por los programas de financiamiento. La mecanización del procedimiento en el seno de las unidades que organizan los cuerpos técnicos, y la fragmentación operativa de la escuela intervenida por partes, reformula los modos en los que se piensa y se practica el proyecto de arquitectura en la estructura estatal sobre el final del siglo XX.

Tras la crisis que dio origen al siglo XXI y la reorganización institucional de 2003 , se inicia un proceso de reactivación de la obra pública que protagoniza el Programa Nacional 700 Escuelas (PN700E) destinado a la construcción de edificios nuevos y al reemplazo de edificios obsoletos.

El marco legislativo que le da sustento al PN700E está determinado por la Ley Nacional de Educación (2006) que, aunque evade el tema de la arquitectura escolar, define políticas públicas en materia educativa y social que tienen consecuencias en las decisiones proyectuales, por ejemplo: al definir como prioridad a los sectores sociales más vulnerables, la mayoría de las obras se localizan en situaciones urbanas comprometidas. 


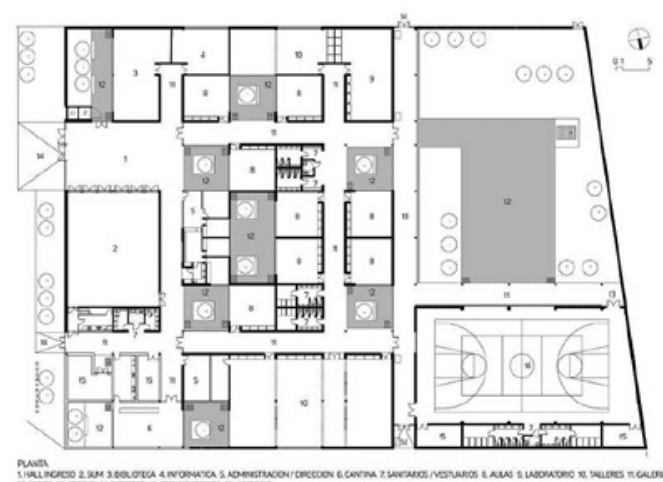

Figura 10 Escuela de Enseñanza Técnica 508, planta, 2008-11, UPE, MOPyV, Santa Fe. Fuente: Mario Corea Arquitectura. En http://mariocorea.com/obras/docente/unidad-de-proyectosespeciales-santa-fe/

El programa consta de tres manuales que constituyen un marco conceptual y procedimental para la producción de arquitectura escolar nacional entre 2004 y $2015^{7}$. Los principales criterios de diseño del programa son: sugerencia de esquemas básicos diseñados según seis zonas bioclimáticas que dividen el territorio nacional, en la que evita definiciones sobre el lenguaje arquitectónico; uso extracurricular de locales escolares (biblioteca y sala de informática, talleres, SUM y espacios exteriores), que establece especificaciones técnicas y de localización dentro del conjunto escolar; y determinación de la Zona Escolar (sistema de dispositivos de señalización y comunicación que alerta sobre la proximidad de una escuela).

Los criterios de diseño expuestos en los manuales actúan en simultáneo con los Criterios y Normativa Básica de Arquitectura Escolar (1998) y tienen resultados diferentes en cada una de las provincias. Ahora bien, a diferencia de los programas nacionales de fin de siglo $X X$, cuyo énfasis estaba puesto en aspectos técnicos $y$ procedimentales, con un consecuente retraimiento del valor simbólico de la arquitectura pública, lo novedoso del PN700E es que los manuales ponen el acento en algunas definiciones de carácter político acerca de la arquitectura escolar, como la no neutralidad de las decisiones proyectuales o la necesaria interrelación entre proyecto educativo y proyecto social del programa de referencia. Este fortalecimiento del rol político de las decisiones en materia disciplinar es un punto de contacto entre el programa nacional y el plan de escuelas propuesto por el gobierno santafesino en 2008.

En 2007, en Santa Fe, se crea la Unidad de Proyectos Especiales (UPE), oficina de proyectos de funcionamiento transversal a las áreas temáticas, que presenta un plan en el que la escuela aparece como programa subordinado a la retórica común de toda la obra pública.

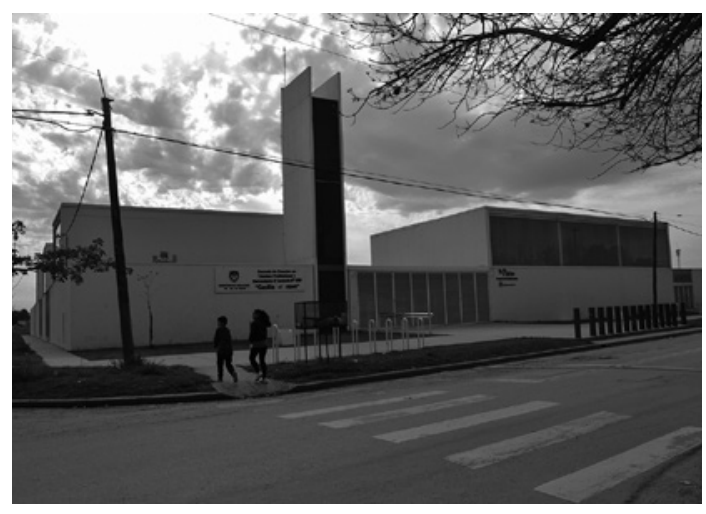

Figura 11 Escuela de Enseñanza Técnica 508, fachada principal, 2008-11, UPE, MOPyV, Santa Fe. Fotografía: Lucía Espinoza

La metodología propuesta para resolver los proyectos de todos los niveles educativos es el Sistema Tipológico Proyectual diseñado por el equipo técnico de la UPE conducido por el arquitecto Mario Corea como asesor (UPE, 2009; Corea, 2013). El método produce revisiones sobre fórmulas y constantes del espacio escolar tradicional. Desde este enfoque, interesa valorar algunos de los elementos constitutivos de la propuesta: el empleo de elementos simbólicos y referencias urbano-arquitectónicas; la organización no jerárquica del programa escolar; el agrupamiento por sectores funcionales; el uso extracurricular de locales escolares y la cualificación de los locales; la localización equidistante de los baños en la trama; la cota homogénea del conjunto escolar y la flexibilidad interior (Figuras 10 y 11).

Simultáneamente, y como aspectos que retoman la premisa pedagógica del "encierro", se propone una vez más en la historia disciplinar, un planteo introspectivo de edificio escolar que redefine el problema de la discontinuidad entre el edificio y la ciudad. La propuesta final se traduce en un prototipo arquitectónico cuya flexibilidad radica en los movimientos endógenos que posibilita la trama cerrada definida por el sistema. Aún más, la sectorización propuesta entre los espacios exclusivos de uso escolar y los locales pensados para ser compartidos con los programas culturales en contra turnos, se delimitan con ajustada precisión en el planteo, manifestando una estructura de máximo control sobre el organismo arquitectónico (Figuras 12 y 13).

Por otra parte, el proyecto resultante de la aplicación del dispositivo metodológico, evidencia el estado de crisis de algunos supuestos disciplinares históricos sobre la obra pública, entre ellos: la transparencia del programa en el lenguaje arquitectónico; la relación de la obra con el entorno inmediato; la actuación profesional desde el aparato burocrático, o el valor del proyecto por concurso en la obra pública. 


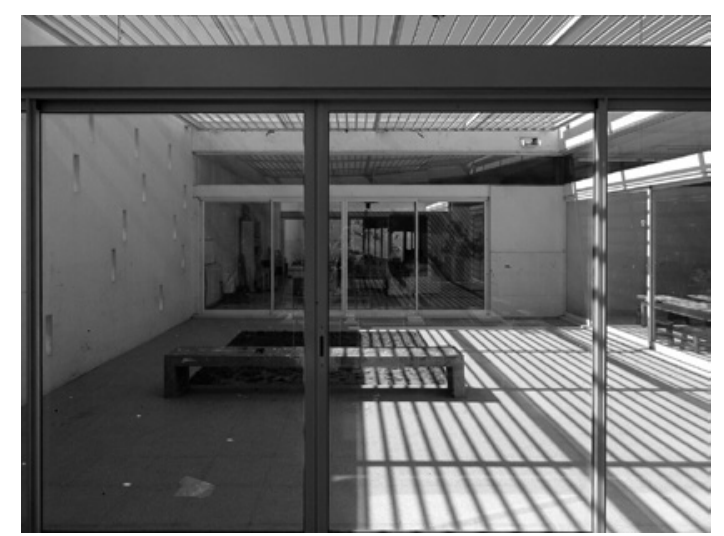

Figura 12 Escuela de Enseñanza Técnica 508, patio interior - expansión aula, 2008-11, UPE, MOPyV, Santa Fe. Fuente: Archivo Cátedra Teoría y Crítica, FADU, UNL

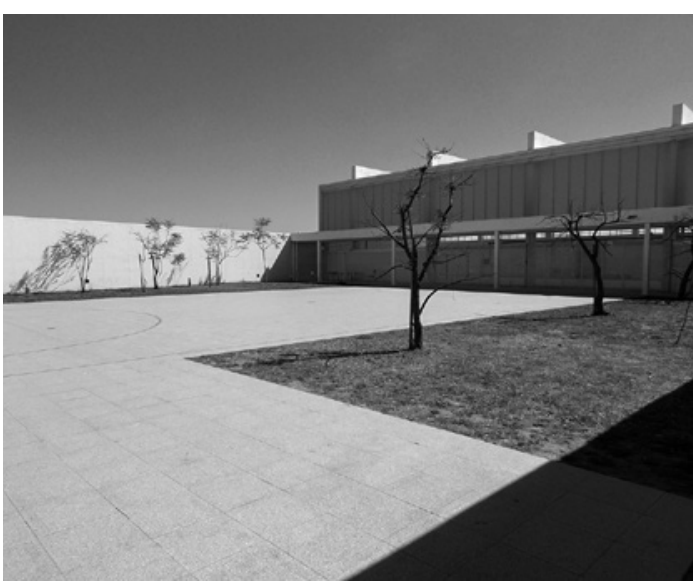

Figura 13 Escuela de Enseñanza Técnica 508, patio común, 2008-11, UPE, MOPyV, Santa Fe. Fuente: Archivo Cátedra Teoría y Crítica, FADU, UNL.

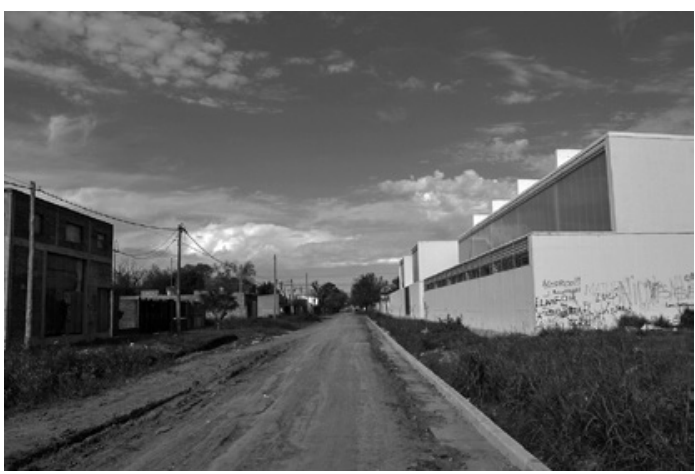

Figura 14 Escuela de Enseñanza Técnica 508, fachada lateral (norte), 2008-11, UPE, MOPyV, Santa Fe. Fotografía: Lucía Espinoza
El proyecto escolar resultante tiene una fórmula que se muestra conveniente para las demandas del escenario comunicacional contemporáneo, ya que está basado en la abstracción, la homogeneidad cromática, la repetición de componentes arquitectónicos y su combinación, con el fin de lograr una imagen identificadora del modelo. Además, su multiplicación en todos los niveles educativos da como resultado una pieza arquitectónica reconocible, que se distingue en los emplazamientos urbano marginales o rurales en los que se localiza ( $\mathrm{Fi}$ gura 14).

La propuesta cobra importancia en una doble dimensión histórico disciplinar, es decir, atravesada tanto por los problemas específicos que recorre la historia de los edificios escolares en Argentina, como por las condiciones que tensionan al proyecto arquitectónico en la contemporaneidad.

\section{CONCLUSIONES}

Si bien, desde el inicio del sistema educativo nacional, la arquitectura ha sido considerada una componente constitutiva, los modelos arquitectónicos, las dimensiones sugeridas y el equipamiento han sido, efectivamente, elementos previos a la discusión sobre los planes y los proyectos desencadenadas en el interior de las oficinas técnicas con la conformación de la estructura estatal moderna, y se han incorporado bajo justificaciones de índole pedagógica o razones de políticas educativas, como por ejemplo, la elección de las referencias a los modelos del palacio o del templo.

Esta primera plataforma conceptual y metodológica basada en la instrumentalización de la arquitectura, que determina modalidades de producción a partir de la formación de equipos técnicos en oficinas y también con contrataciones directas desde el inicio del sistema, establece estrategias de abordaje del edificio escolar como problema proyectual en el cual el organismo arquitectónico concentra las definiciones pedagógicas y políticas de la propuesta.

Promediando el siglo XX, el Primer Plan Quinquenal introduce un giro conceptual respecto de la obra pública, como componente de un plan y en relación al resto de los dispositivos que integran el sistema de acción social. Otro hito del periodo es la incorporación de la perspectiva regional, tanto en la concepción del edificio y en la expresión arquitectónica, como en su programa pedagógico, que concentra las definiciones en los organismos nacionales.

No obstante, este trabajo pone la lupa sobre la operación reformista que cierra el siglo XX porque ésta se ejecuta sobre una metodología compleja (fragmentadora, interdisciplinaria, exigente en requisitos técnicos y mediada por programas, normativas y manuales) para proyectar el edificio escolar dentro de la estructura estatal, capitalizando la experiencia acumulada y la eficiencia del aparato burocrático con miras a reproducir una nueva modalidad operativa que se funde con las formas instaladas. 
En consecuencia, si bien el PN700E sostiene la perspectiva regional en el planteo de prototipos desde aspectos compositivos y tecnológicos, también propone descartar la opción de un lenguaje homogéneo, que facilitaría la visibilidad de la obra de gobierno, en favor de la búsqueda de una "unidad conceptual del programa" (Trlin, 2007: 46) que quedaría determinada por las pautas fijas enunciadas en los tres manuales operativos. Esta decisión de refuerzo sobre las autonomías provinciales deriva en que las definiciones sobre arquitectura descansan en proyectos elaborados desde cada una de las jurisdicciones subnacionales: desde las modalidades de participación profesional hasta las medidas sobre arquitectura escolar.

A modo de ejemplo, mientras Santa Fe conforma un equipo técnico destinado a diseñar un lenguaje homogéneo para toda la obra de una gestión, Córdoba, bajo el mecanismo de la contratación de profesionales externos a la estructura técnica estatal y por la vía de la mediación institucional, desarrolla un sistema proyectual para edificios concebidos como Anexos, destinados al polimodal (Santocono, 2013).

En definitiva, el PN700E y sus manuales operativos constituyen un marco conceptualmente tensionador y procedimentalmente reestructurador de la producción de arquitectura escolar desarrollada pero, al mismo tiempo, se verifican resultados diferentes entre las propuestas de cada jurisdicción que dan cuenta de relaciones y objetivos distintos entre las políticas públicas en educación y en arquitectura.

Finalmente, si, por un lado, las condiciones de producción que determinan el inicio del siglo XXI reconocen un origen en las transformaciones ejecutadas en el pasado reciente, por otro, la producción de arquitectura escolar contemporánea da cuenta de cierta continuidad histórica en el carácter instrumental asignado a la disciplina, renovando tensiones entre arquitectura y política a partir de la confluencia de nuevas e históricas determinaciones para la arquitectura pública de gestión estatal.

Desde la decisión de trabajar con un esquema tipológico asociado a un programa o nivel educativo, hasta el diseño de un prototipo que asuma todos los programas escolares en cualquier situación ambiental o cultural, la relación entre los proyectos que propone la arquitectura y los proyectos diseñados por la pedagogía es variable en la historia: se encuentran, se superponen y también se distancian en un devenir en el que intervienen las políticas públicas nacionales, subnacionales y sus articulaciones.

En el prolífico contexto de convivencia de programas de infraestructura escolar diseñados en los países de América Latina que revisan las modalidades tradicionales de participación profesional, las técnicas constructivas en relación a los recursos productivos, el capital de la arquitectura frente a la ciudad fragmentada, pero también las prácticas proyectuales asumidas como relacionales entre la arquitectura escolar y la pedagogía ${ }^{8}$, se impone en Argentina retomar la reflexión sobre el vínculo entre arquitectura, políticas públicas y la propia historia entre ambos campos de actuación con el fin de revisar aquellas dimensiones establecidas como certezas.

[8] Programas PICE (San Pablo), en Brasil; PECEC (Medellín) Megacolegios (Bogotá), Centros de Desarrollo Infantil del Programa de Cero a Siempre del Gobierno Nacional, en Colombia; y PAEPU, en Uruguay.

\section{REFERENCIAS BIBLIOGRÁFICAS}

BRANDARIZ, Gustavo. La arquitectura escolar de inspiración sarmientina. Buenos Aires: Eudeba, 1998.

CATTANEO, Daniela. La arquitectura escolar como instrumento del Estado. Contrapuntos Nación-provincias en la década de 1930 [en línea]. Colección tesis doctorales, FAPyD-UNR, Rosario: A\&PUNR Editora, 2015. Disponible en http://www.fapyd.unr.edu.ar/ ayp-ediciones/tesis-doctorales/

COREA, Mario. Las escuelas de Santa Fe. Buenos Aires: NobukoSCA, 2013.

DURÁ GÚRPIDE, Isabel. La construcción de escuelas en Mendoza durante el primer peronismo (1946-1955). La acción complementaria del gobierno nacional, la Fundación Eva Perón y los gobiernos provinciales. En: XVI Jornadas Interescuelas, Departamentos de Historia, Mar del Plata, 2017.

ESPINOZA, Lucía. Arquitectura escolary Estado moderno. Santa Fe 1900-1943, Polis Científica, vol. 6. Santa Fe: UNL, 2005.

ESPINOZA, Lucía, Escuelas del cincuenta. Reflexiones sobre la relación Arquitectura y Estado en la Argentina peronista. En: Actas del $9^{\circ}$ Seminario DOCOMOMO Brasil Interdisciplinaridade e experiências em documentação e preservação do patrimônio recente, Brasilia, Brasil, junio de 2011.

ESPINOZA, Lucía. Arquitectura educativa y políticas públicas en Santa Fe (2007-2011). Producción y comunicación de la arquitectura en el Estado [en línea]. Colección tesis doctorales, FAPyD-UNR, Rosario: A\&P-UNR Editora, 2016. Disponible en http://www. fapyd.unr.edu.ar/wpcontent/uploads/2016/05/tesis_espinoza. pdf

GIZZARELLI, Marcelo H. La pequeña utopía urbana. Escuelas municipales 1880/1930. Summarios, 1985, vol. 91/92, Buenos Aires, pp. 14-22

GORELIK, Adrián. Ciudad, modernidad y modernización [en línea]. Universitas Humanística, 2003, vol. 56, n 56, Disponible en http://revistas.javeriana.edu.co/index.php/univhumanistica/ article/view/9694

MINISTERIO DE CULTURA YEDUCACIÓN DE LA NACIÓN. Criterios y Normativa Básica de Arquitectura Escolar. Buenos Aires, 1998.

MURILLO DASSO, María Florencia. La arquitectura escolar en San Miguel de Tucumán, siglos XIX al XXI. Tesis de Magister inédita. Facultad de Arquitectura y Urbanismo de la Universidad Nacional de Tucumán, Tucumán, 2012.

RAFFA, Cecilia y ESPINOZA, Lucía. Patrimonio reciente. Aportes históricos para la valoración cultural de la arquitectura pública en dos ciudades argentinas: Santa Fe y Mendoza (1932-1943). En: Arquimemoria 4. La dimensao urbana do patrimônio. Actas del Arquimemoria 4. Salvador-Bahia, Brasil: Faculdade de Arquitetura (UFBA), 2013.

SANTOCONO, Ricardo (coord.) Programa Nacional 700 Escuelas. $1^{\text {era }}$ ed. Ciudad Autónoma de Buenos Aires: Ministerio de Planificación Federal, Inversión Pública y Servicios, Presidencia de la Nación, 2013.

SHMITD, Claudia. Escuela. En: LIERNUR, Francisco y ALIATA, Fernando (comp.). Diccionario de Arquitectura en Argentina. T. E-H. Buenos Aires: Clarín Arquitectura, 2004, pp. 44-50.

TRLIN, Margarita. Programa Nacional 700 Escuelas. Summa + Educación, 2007, n 90, p. 46.

UNIDAD DE PROYECTOS ESPECIALES (UPE). Santa Fe En Obras, 2008-2009. Santa Fe: Ministerio de Obras Públicas y Vivienda de la Provincia de Santa Fe, 2009. 